\title{
Measurement of fracture toughness by nanoindentation methods: recent advances and future
}

\section{challenges}

\author{
M. Sebastiani*1 ${ }^{1}$, K. E. Johanns ${ }^{2}$, E. G. Herbert ${ }^{3}$, and G. M. Pharr ${ }^{3,4}$ \\ ${ }^{1}$ Roma TRE University, Engineering Department, Italy \\ ${ }^{2}$ Department of Materials Science, TU Darmstadt, D-64287 Darmstadt, Germany \\ ${ }^{3}$ Department of Materials Science \& Engineering, The University of Tennessee, Knoxville, TN, \\ USA \\ ${ }^{4}$ Materials Science and Technology Division, Oak Ridge National Laboratory, Oak Ridge, TN, USA
}

*corresponding author: marco.sebastiani@uniroma3.it

\begin{abstract}
In this paper, we describe recent advances and developments for the measurement of fracture toughness at small scales by the use of nanoindentation-based methods including techniques based on micro-cantilever, beam bending and micro-pillar splitting. A critical comparison of the techniques is made by testing a selected group of bulk and thin film materials. For pillar splitting, cohesive zone finite element simulations are used to validate a simple relationship between the critical load at failure, the pillar radius, and the fracture toughness for a range of material properties and coating/substrate combinations. The minimum pillar diameter required for nucleation and growth of a crack during indentation is also estimated. An analysis of pillar splitting for a film on a dissimilar substrate material shows that the critical load for splitting is relatively insensitive to the substrate compliance for a large range of material properties. Experimental results from a selected group of materials show good agreement between single cantilever and pillar splitting methods, while a discrepancy of $\sim 25 \%$ is found between the pillar splitting technique and double-cantilever testing. It is concluded that both the micro-cantilever and pillar splitting techniques are valuable methods for micro-scale assessment of fracture toughness of brittle ceramics, provided the underlying assumptions can be validated. Although the pillar splitting method has some advantages because of the simplicity of sample preparation and testing, it is not applicable to most metals because their higher toughness prevents splitting, and in this case, microcantilever bend testing is preferred.
\end{abstract}

Keywords: fracture toughness, nanoindentation, cantilever, pillar, micron-scale 


\section{Introduction}

Detailed characterization of the mechanical behavior of thin films and small-scale devices is of paramount importance in understanding their in-service failure mechanisms. To this end, nanoindentation has been widely used in the last two decades as a high spatial resolution microprobe for measuring mechanical properties of materials at small scales [1-2]. Among the mechanical properties that can be quantitatively evaluated by this technique are: hardness and elastic modulus [1-2], storage and loss modulus [3], strain rate sensitivity [4], yield strength and strain hardening coefficient [5], residual stress [6], adhesive strength of coatings [7] and fracture toughness [8-27].

Numerous methods exist for the measurement of fracture toughness of small volumes of material. Indentation based methods with sharp pyramidal indenters have been widely investigated [8-15] due to the relative ease of testing and sample preparation. In such methods, the fracture toughness is determined from measurements of the lengths of cracks emanating from the residual indentation impression at a given load (Fig. 1). The choice of the model for determining the indentation fracture toughness depends on the type of crack system, e.g., median, radial, half-penny, cone, or lateral cracks [16-17], and the geometry of the pyramidal indenter. Generally, equations relating fracture toughness to applied loads and crack lengths from pyramidal indentation tests have the form of [16-17]:

$$
K_{c}=\frac{P_{\max }}{c^{3 / 2}} \prod\left(\frac{E}{H}, v, \psi, \frac{c}{a}\right),
$$

where $\boldsymbol{K}_{\boldsymbol{c}}$ is the fracture toughness, $\boldsymbol{P}_{\max }$ is the maximum indentation load, $\boldsymbol{c}$ and $\boldsymbol{a}$ are the crack length and the contact size (the distance from the center to the indentation to the corner of the contact), respectively, $\boldsymbol{E}$ is the elastic modulus, $\boldsymbol{H}$ is the hardness, $\boldsymbol{v}$ is the Poisson's ratio, and $\boldsymbol{\psi}$ is the axis-to-face angle of the pyramidal indenter. It is clear from this equation that the selection of an appropriate model for indentation fracture toughness requires a knowledge of the elastic and plastic properties of the material, the lengths of the cracks, and the indenter geometry. A simplified analysis can be performed in the case of long cracks $(\boldsymbol{c} / \boldsymbol{a}>>1)$, which leads to the classic LawnEvans-Marshall (LEM) model [8-9]:

$$
K_{c}=\alpha \cdot \sqrt{\frac{E}{H}} \frac{P_{\max }}{c^{3 / 2}} .
$$

The value of the LEM coefficient, $\boldsymbol{\alpha}$, has been experimentally calibrated over a number of bulk, brittle materials and found to be $\sim 0.016$ for the Vickers 4 -sided pyramidal indenter. However, recent studies of indentation cracking with cohesive finite element calculations [16-17] have shown that $\boldsymbol{\alpha}$ depends significantly on the ratio of $\boldsymbol{E} / \boldsymbol{H}$, Poisson's ratio, and indenter geometry. This is because median type cracking dominates at low $\boldsymbol{E} / \boldsymbol{H}$ ratios and Palmqvist type cracking at higher 
ratios [17], and this changeover in mechanism has significant implications for how the toughness is related to the crack length. Consequently, the choice of the most appropriate model for toughness evaluation from radial crack measurements is complex. In addition, the LEM model requires accurate measurements of crack lengths, which can sometimes be difficult at small length scales. Critical issues associated with indentation cracking methods based on observations from cohesive zone finite element simulations have recently been discussed in detail [16,17].

In case of thin films, the measurement of fracture toughness is made even more complex by influences of the substrate, which may enhance or inhibit plastic zone development and crack development, especially as the size of the contact approaches the thickness of the film. In addition, the large residual stresses that can exist in thin films can substantially alter the cracking behavior, making the use of indentation-based techniques unsuitable in practice unless the residual stresses are precisely known by other methods [26]. The establishment of a correlation between measured quantities and actual in-service failure modes is even more complicated, since it arises from a complex interaction between the intrinsic resistance to fracture and the residual stress field present in the film [26]. Microstructural features (e.g., grain size and distribution, defect density, and the substrate/coating interface) further contribute to the complexity of the problem.

A new class of techniques has recently been developed to resolve some of the issues associated with indentation based fracture toughness measurements of films. The methods generally use a nanoindentation system to apply force to and measure the displacements of micro-scale mechanical test specimens of various geometry produced by focused ion beam (FIB) milling.

The specimen geometries used in these tests include single cantilever beams [18; 20-22; 49-50], clamped beams [19; 27], double cantilever beams [23], membranes [24-25], and pillars [26]. In case of the single-cantilever beam specimens, a pre-notched micro-specimen is deformed in bending until crack propagation is induced, as shown in Fig. 2. An analytical model is then used to calculate the fracture toughness from measured values of the critical loads for crack extension, crack lengths, and specimen sizes using:

$$
K_{c}=\sigma_{c} \cdot \sqrt{\pi a} \cdot F\left(\frac{a}{b}\right),
$$

where $\sigma_{c}$ is the fracture stress, $\boldsymbol{a}$ is the crack length and $\boldsymbol{F}(\boldsymbol{a} / \boldsymbol{b})$ is a dimensionless shape factor that depends on sample geometry, which is illustrated in Fig. 2 [18]. In addition to monolithic materials and thin films, cantilever-bending methods have been used to test the interfacial toughness of grain boundaries [21] and the adhesion of coatings [22].

Double cantilever beam testing at small scales can be conducted in a simple compression experiment using a specimen with a special geometry like that shown in Fig. 3 [23]. Such tests are typically performed in situ (i.e., inside a scanning electron microscope) on FIB-milled, pre-notched 
double-cantilever beams, like that shown in Fig. 3. The fracture toughness of such specimens is evaluated from the compression load at crack extension using:

$$
K_{c}=\sqrt{3} \frac{(e-\mu h)}{l d^{3 / 2}} P_{c}
$$

where $\boldsymbol{l}$ is the width of the specimen, $\boldsymbol{e}$ is the distance between the line of action of the load $\boldsymbol{P}$ and the neutral axis of the beam, $\boldsymbol{P}_{\boldsymbol{c}}$ is the critical load for crack extension and $\boldsymbol{\mu}$ is the friction coefficient between the flat punch indenter, used to apply the compression to the specimen [23]. Multiple load-unload cycles are usually performed in order to study the influences of friction and plasticity on the toughness measurements. One of the drawbacks of cantilever-based methods is that specimen preparation by FIB milling may induce structural damage, especially at the root of the stress-concentrating notch, where it may influence the crack propagation load. In addition, for the double cantilever method, a tedious calibration procedure is required to estimate the friction coefficient between the indenter and the specimen. In a research environment, the impact of these issues can usually be minimized, but they may pose significant obstacles for industrial application in which the techniques are often used for quality control purposes that must be simple and fast.

In order to avoid some of these issues, a new, relatively simple method for measuring the fracture toughness at small scales has been recently developed based on sharp indentation testing of FIB milled micro-pillars [26]. The basic principle is that sharp indentation of FIB milled micropillars of specific geometry results in fracture by splitting (see Fig. 4) at reproducible loads that are readily quantified from displacement bursts in the loading segment of the load-displacement curve [26]. The method retains some of the advantages of the conventional indentation fracture toughness approach in terms of test simplicity, but eliminates two of its major drawbacks: (1) having to accurately image and measure the indentation crack lengths, and (2) when carefully executes, it can eliminate the effects of film stress on the measurements. In this method, fracture toughness is computed from a simple relationship between the critical load at failure, $\boldsymbol{P}_{\boldsymbol{c}}$, the pillar radius, $\boldsymbol{R}$, and the elastic and plastic properties of the material [26] through:

$$
K_{c}=\gamma \frac{P_{c}}{R^{3 / 2}},
$$

where the dimensionless coefficient $\boldsymbol{\gamma}$ is different from the LEM coefficient, $\boldsymbol{\alpha}$. Note that the coefficient $\boldsymbol{\gamma}$ includes the influences of elastic-plastic properties and is therefore material specific. The utility of Eq. 5 lies in its simplicity, as both the critical load and pillar radius are easily measured quantities. In addition, knowledge or measurement of the crack length and geometry is not required, even though it may be possible to measure through post-mortem SEM analysis of the 
pillars. The main drawback is that the coefficient $\gamma$ is unknown and must be evaluated with numerical techniques. This disadvantage, among others, will be discussed in the next section.

Equation (5) was initially derived by a simple dimensional argument, taking into account the primary variables of interest (fracture toughness, instability load, and pillar radius) [26]. A more complete description of failure during the pillar splitting test would include detailed consideration of the stress intensity factor and important geometrical effects like the ratio of crack length to pillar radius, which is not directly accounted for in Eq. 5 [16-17]. From an experimental viewpoint, where only the pillar radius and instability load are known, Eq. 5 facilitates the estimation of fracture toughness provided $\boldsymbol{\gamma}$ for a given material has been calibrated with finite element simulations. In addition to its simplicity, the pillar splitting test has some additional advantages that make it attractive for toughness assessment of thin coatings and small-scale devices. Specifically, measurement of crack lengths or fracture surfaces after the test is not required, although doing so may help confirm that unstable crack propagation was the source of the observed instability. In addition, since the residual stress in the upper portion of the pillar is totally relaxed when the aspect ratio (height to diameter) is greater than 1.0 [35], residual stresses in the coating have little influence on the toughness measurement. Substrate induced artifacts are also reduced since pillar splitting can occur at shallow indentation depths, and the influences of FIB damage are expected to be less significant because the FIB damage is surface-localized in a volume that is relatively far from the position of crack nucleation and propagation. However, the pillar splitting technique is currently limited by the requirement of cohesive zone finite element techniques for any specific material under investigation. In this context, a more comprehensive analysis of the gamma coefficients as a function of the $\boldsymbol{E} / \boldsymbol{H}$ ratio is required.

To that end, new cohesive finite element simulations are performed in this work in order to provide the necessary relationship between the fracture toughness, critical load and pillar radius for a wide range of materials, including ceramics and metals.

An additional focus of this work is to provide a quantitative comparison of the different methods in order to examine key differences and provide guidelines on which methodology may be best for a given application. To achieve this, four different materials were selected for experimental testing: (1) a (100) single-crystal silicon sample; (2) a chromium nitride ( $\mathrm{CrN}$ ) coating produced by cathodic arc physical vapor deposition techniques (CAE-PVD) [23]; (3) a CrN coating produced by magnetron sputtering PVD [20]; and a (4) bulk metallic glass (BMG). All of the samples were measured by pillar splitting, and two of the PVD CrN coatings were examined by single and double-cantilever tests for direct comparison. 


\section{Finite element and approximate analytical modeling of pillar splitting experiments}

\subsection{Calibration of the method for a wide range of material properties}

In a previous description of the pillar splitting test, a non-dimensional analysis was adopted to relate the critical stress intensity factor to the applied indenter loads and sample geometry [26]. Validation of Eq. (5) was performed for a small set of materials, namely $\mathrm{TiN}, \mathrm{CrN}$ and a $\mathrm{CrAlN} / \mathrm{Si}_{3} \mathrm{~N}_{4}$ nanocomposite [26]. The finite element software package ABAQUS-v6 [25] was used in three-dimensional simulations of a rigid, Berkovich pyramidal indenter (centerline-to-face angle of $65.3^{\circ}$ ) contacting an isotropic, elastic-perfectly plastic, (i.e., no work hardening) cylindrical pillar (see Fig. 5), with cohesive elements aligned along each of the indenter edges in a direction perpendicular to the free (indented) surface. A MAXS criterion (maximum stress) was used for the onset of debonding, and a fracture energy was used for crack nucleation [28]. Crack growth was dictated by material properties, loading conditions, and pillar geometry but was constrained to remain within the defined crack plane, noting that crack growth in experiments may have more complex geometries. In all cases, the cohesive elements were utilized in a manner that resulted in brittle cracking behavior that could be described by linear-elastic fracture mechanics in the limit that the crack length was at least ten times greater than the cohesive zone size [31-32]. Additional details of the FEM procedures and boundary conditions are reported elsewhere [26].

Here, we further investigate the validity of Eq. (5) by exploring a wider range of materials (in terms of variation of the $\boldsymbol{E} / \boldsymbol{H}$ ratio), and effects of substrate compliance for the case of a pillar on a dissimilar substrate. The simulations were used to estimate instability loads as a function of pillar radius and fracture toughness for materials with elastic modulus to hardness ratio $(\boldsymbol{E} / \boldsymbol{H})$ ranging from 5 to 30. Although Poisson's ratio was fixed at 0.25 , we note that Lee et al. [16] have observed an increase in the LEM coefficient $\alpha$ in Eq. 2 for decreasing values of Poisson's ratio. A similar Poisson's ratio dependence may be expected for the pillar splitting test since the origin of crack driving forces is the same. However, this was not explicitly explored in this study.

Fig. 6 presents load-displacement curves for one representative brittle coating on 3 different substrates, where displacement into surface, $\boldsymbol{h}$, refers to the total displacement of the tip of the indenter from the initial point of contact. The load-displacement behavior resembles the elasticplastic indentation of bulk materials until a sharp load drop occurs at a critical point. This is consistent with the experimental behavior with the caveat that the experiments were performed in load control, wherein a displacement burst is observed, while the simulations were performed under displacement control, so a sharp load drop is observed. As demonstrated in a previous study [26], the load drop occurs consistently at a unique value of $\boldsymbol{P} / \boldsymbol{K}_{c} \boldsymbol{R}^{3 / 2}$ for a given material. 
Crack geometries predicted by the finite element simulations just before and just after the critical instability load, $\boldsymbol{P}_{\boldsymbol{c}}$, for the same representative material are pictured in Fig. 7. Even though the FEM solution converged, the load drop occurred rapidly, and the use of viscous regularization drastically changed the subsequent cohesive element behavior [33]. Thus, the geometry of the crack post load drop is not consistent with the fracture toughness specified in the simulation. That being said, the crack geometries up to and at the critical instability load are consistent with the input value of fracture toughness. It should also be noted that the finite element simulations naturally account for cracks that nucleate near the surface in a Palmqvist-like fashion or beneath the surface in the way that median/radial cracks would form. Which predominates depends on the elastic and plastic properties of the material [17].

In previous work [26], we estimated $\boldsymbol{\gamma}$ for three specific ceramic coating materials: TiN, $\mathrm{CrN}$, and $\mathrm{CrAlN}-\mathrm{Si}_{3} \mathrm{~N}_{4}$. To facilitate the measurement of fracture toughness for other hard ceramic materials with the pillar splitting technique, Fig. 8 includes additional finite element results for values of $\boldsymbol{\gamma}$ over an $\boldsymbol{E} / \boldsymbol{H}$ range from 5 to $\sim 30$, all for a Berkovich indenter and a Poisson's ratio of 0.25. Some values of the elastic modulus to yield strength ratio, $\boldsymbol{E} / \boldsymbol{Y}$, used in the simulations are provided on the plot. The ranges of $\boldsymbol{K}_{\boldsymbol{c}}(2$ to $9 \mathrm{MPa} \sqrt{\mathrm{m}})$ and $\boldsymbol{E} / \boldsymbol{H}$ that have been investigated are representative of a wide range of ceramic materials. Limitations of the method when applied to metals will be discussed later in this paper. As shown in Fig. 8, the average values of the coefficient $\boldsymbol{\gamma}$ (Eq. 5) range from as low as 0.145 for materials like fused silica $(\boldsymbol{E} / \boldsymbol{H} \approx 7.0$ ) up to 0.4 for materials with an $\boldsymbol{E} / \boldsymbol{H}$ value greater than 30.0. It is important to note that the data reported in Fig. 8 apply only to a Poisson's ratio of 0.25 . Minor modifications of the gamma coefficient are expected for different Poisson's ratio values.

\subsection{Effects of substrate compliance on the pillar splitting technique}

Substrate compliance is generally important when measuring the hardness and modulus of a film or coating from instrumented indentation data [42]. The substrate can influence elastic-plastic deformation in the film and thus the crack driving forces. There can also be interactions between the crack tip and the substrate. To address the influences of substrate compliance on the pillar splitting technique, we carried out additional simulations with compliant substrates having a elastic modulus of $\boldsymbol{E}_{s}$ and a Poisson's ratio of 0.25. The influences of substrate compliance on $\gamma$ are included in Fig. 8 for direct comparison to the rigid substrate results discussed in the previous section. This dataset is not to be viewed as a comprehensive description of substrate effects, but rather to show that while substrate compliance is important, the overall change in $\gamma$ from a rigid to a compliant one with a modulus two orders of magnitude less than the modulus of the pillar is well within experimental scatter in data. 
Load-displacement curves for a pillar on different substrates are plotted in Fig. 6 to provide further information on the influence of substrate compliance. Here, the pillar material has an elastic modulus to yield strength ratio of 30 , a Poisson's ratio of 0.25 , a fracture toughness of $1.93 \mathrm{MPa}$ $\mathrm{m}^{1 / 2}$, and the pillar has a radius of $150 \mu \mathrm{m}$. A rigid substrate, steel substrate with an elastic modulus of $200 \mathrm{GPa}$, and a polymethyl methacrylate (PMMA) substrate with an elastic modulus of $2 \mathrm{GPa}$ were simulated. While the indenter displacement is dramatically influenced by substrate compliance, the instability load, $\boldsymbol{P}_{\boldsymbol{c}}$, is largely unaffected, with only an $\sim 11 \%$ difference in the load at failure between the rigid and polymer substrates. Note that the failure loads and pillar radius modeled in Fig. 6 are large compared to experimental conditions, where failure loads are typically on the order of $\sim 10 \mathrm{mN}$ and pillar radii are on the order of $\sim 5 \mu \mathrm{m}$. The reason for the large loads and radii in the finite element simulations is due to the formulation of the cohesive zone constitutive behavior and the small-scale yielding criterion [16-17]. It should be noted that once unstable crack growth commences, the behavior of the system will be dependent on the dynamics of the testing instrument and compliance of the substrate. Thus, there may be a condition wherein the critical load is not easily defined, since a very compliant substrate or testing system may damp the instability.

\subsection{Approximation of the basic equation for the pillar splitting technique}

In order to provide a semi-theoretical basis for the relation proposed in Eq. (5), we start with a simple expression for the stress intensity factor of a semi-elliptical surface crack to derive the point of unstable crack growth in the pillar splitting test. In its simplest form, the mode-I stress intensity factor, $\boldsymbol{K}_{\boldsymbol{I}}$, at the crack tip of a centrally loaded, semi-elliptical crack in a semi-infinite, homogeneous solid is given by:

$$
K_{I}=A \frac{F}{c^{3 / 2}},
$$

where $\boldsymbol{F}$ is the applied force, $\boldsymbol{c}$ is the crack length at the surface, and the coefficient $\boldsymbol{A}$, which accounts for free surface effects, is a function of crack-tip position relative to the free surface [36]. When the semi-elliptical surface crack is in a specimen with pillar geometry, the stress intensity factor is magnified as the crack tip approaches the free surface, i.e., the radius of the pillar $\boldsymbol{R}$. Shah and Kobayashi [38] introduced a stress intensity magnification factor, $\boldsymbol{M}_{\boldsymbol{f}}$, to modify the semiinfinite stress intensity factor of a semi-elliptical crack approaching a free surface. We make use of their result by assuming that:

- the magnification factor has the same functional form $\boldsymbol{M}_{f}=(\boldsymbol{1}-\boldsymbol{c} / \boldsymbol{R})^{-1}$.

- the crack is in equilibrium just before failure, i.e., the stress intensity factor is equal to the fracture toughness, $\boldsymbol{K}_{I \boldsymbol{c}}$, of the material. 
- the material does not exhibit rising R-curve behavior as the crack grows to its length at failure during the pillar splitting test.

Incorporating the magnification factor into Eq. 6 yields:

$$
K_{I}=\frac{A}{1 c / R} \frac{F}{c^{3 / 2}} .
$$

Rewriting this equation to express the force, $\boldsymbol{F}$, as a function of crack length, $\boldsymbol{c}$, and taking the derivative with respect to crack length and equating to zero yields a critical crack length for unstable crack growth, $c^{*}$, of $0.60 \cdot \boldsymbol{R}$. This value is in good agreement with observations from the cohesive zone finite element models, although there is some dependence on the elastic modulus to yield strength ratio. Substituting the critical crack length into Eq. 7, replacing the point-force, $\boldsymbol{F}$, with the load on the indenter at failure, $\boldsymbol{P}_{\boldsymbol{c}}$, and combining the constants into the coefficient of proportionality, $\gamma$, produces the relationship in Eq. 5 as deduced previously strictly from dimensional analysis. 


\section{Experimental details}

The samples investigated in this work consisted of: (1) a bulk (100) single-crystal silicon wafer; (2) a $3 \mu \mathrm{m}$ chromium nitride coating ( $\mathrm{CrN}$ ) produced by magnetron sputtering (MS-PVD) [20]; (3) a $9 \mu \mathrm{m}$ thick chromium nitride coating $(\mathrm{CrN})$ produced by cathodic arc evaporation (CAEPVD) [23]; and (4) a Zr-Cu-Ni-Ti-Al bulk metallic glass (BMG) sample. Details of the deposition parameters and other general properties of the coatings are reported elsewhere [20, 23]. The BMG sample was produced by drop casting into a copper mold at room temperature. The diameter of the rod was $\sim 7 \mathrm{~mm}$. The test specimen was obtained by cutting a slice from the original rod, polishing using an automated procedure consisting of a sequence of polishing steps (from 30 to $5 \mu \mathrm{m}$ abrasives), and final polishing for $100 \mathrm{~h}$ with a $0.1 \mu \mathrm{m}$ colloidal silica suspension. Liu et al. independently measured the fracture toughness of the CAE-PVD CrN coating by the doublecantilever bending method [23]. For the MS-PVD CrN coating, the fracture toughness was independently measured by single-cantilever bending tests, as reported in [20]. For comparison purposes, the values obtained by the single and double cantilever experiments are given in Table. 1 . It should be noted that the microstructures of the two $\mathrm{CrN}$ coatings are significantly different, as they were produced by two different PVD methods (arc evaporation and magnetron sputtering). The coatings have different thicknesses and very different grain sizes, as reported in previous papers $[20,26]$. For these reasons, it is not surprising that the fracture toughnesses of the two materials are significantly different.

Fabrication of micro-pillars for the splitting experiments was performed by an optimized focused ion beam (FIB) procedure based on the ring-core milling approach previously developed by one of the authors [34-35]. FIB ring-core milling was performed in a single outer-to-inner pass using a current of $48 \mathrm{pA}$ and an increasing depth of the trench from the outer part of the ring to the inner one. This strategy allows for an optimal reduction of the ion dose at the periphery of the pillar, thereby reducing the influence of ion-induced damage and re-deposition at the edges. The proposed sample geometry is well suited for fracture toughness estimation by pillar indentation splitting because the volume of material where the crack nucleates and propagates, which is located inside the pillar beneath the indentation, is relatively far from the FIB milled surfaces. All pillars were FIB milled to an aspect ratio $(z / D)$ of $\sim 1.0$ and a relative diameter with respect to film thickness $(\boldsymbol{D} / \boldsymbol{t})$ ratio greater than 1.0, where $\mathbf{z}$ is the pillar height, $\boldsymbol{D}$ is the top diameter, and $\boldsymbol{t}$ is the film thickness. According to previous work [34-35], these geometrical conditions result in a complete residual stress relaxation in the upper part of the pillars and minimal effects from substrate compliance during the crack nucleation and propagation, provided the indentation depth is less than $t / \mathbf{1 0}$. It was found by FEM simulation that this aspect ratio assures that the cracks that form remain in the top third of the pillar and thus do not strongly interact with the substrate. The resulting pillar size for 
each material measured by SEM imaging is reported in Table 1. Examples of FIB milled pillars are given in Fig. 9. The procedure developed for micro-pillar FIB milling has been automated to produce more than ten pillars at specific sample location in one overnight session.

In case of the CAE-PVD CrN material, where the pillar diameters were greater than $5 \mu \mathrm{m}$, the pillar indentation splitting tests were performed using an Keysight G200 Nano Indenter equipped with a diamond Berkovich indenter tip and an XP indentation head. The loading was controlled such that the loading rate divided by the applied load was held constant at $0.05 \mathrm{~s}^{-1}$ until unstable propagation of a crack was observed. The continuous stiffness measurement (CSM) technique was not used during these tests. For the MS-PVD CrN coating, where the pillar diameters were $\sim 3 \mu \mathrm{m}$ and positioning accuracy became an issue, an in-SEM nanoindentation device (Nanomechanics Inc.) operated in an FEI Helios Nanolab600 FIB-SEM microscope was used to perform the tests.

The hardness and elastic modulus of the materials were measured by nanoindentation testing [1-2] using a diamond Berkovich indenter tip and an Keysight G200 Nano Indenter. As with the pillar experiments, the magnitude of the loading rate divided by the applied load was held constant at $0.05 \mathrm{~s}^{-1}$. The loading was terminated at a maximum indentation depth of $300 \mathrm{~nm}$. Hardness and elastic modulus were obtained using the CSM technique, as the average of the values measured in the depth range between 100-120 $\mathrm{nm}$. The instrument frame stiffness and indenter area function were carefully calibrated before and after testing on a certified fused silica reference sample. 


\section{Results}

Experimental load-displacement curves representative of all the samples under investigation are shown in Fig. 9, where clear and reproducible displacement bursts corresponding to unstable crack propagation and pillar splitting are observed in all cases. The fracture toughnesses were calculated using Eq. 5 from the average load at failure, the pillar radius, and the value of $\boldsymbol{\gamma}$ found from the cohesive zone finite element simulations. The average instability loads and pillar radii for each material are given in Table 1. Values of the fracture toughness were found to be: $1.16 \mathrm{MPa}$ $\mathrm{m}^{1 / 2}$ for (100) $\mathrm{Si}$; $3.13 \mathrm{MPa} \mathrm{m}^{1 / 2}$ for MS-PVD CrN; and 2.19 MPa $\mathrm{m}^{1 / 2}$ for CAE-PVD CrN. All the experimental results are summarized in Table 1. Literature values for (100) silicon generally fall in the range of 0.9-1.2 $\mathrm{MPa} \mathrm{m}^{1 / 2}$ [48], and are thus consistent with the pillar splitting values. The single-cantilever experiments on the same MS-PVD coating [20] gave a toughness of 3.10 MPa $\mathrm{m}^{1 / 2}$, which is in good agreement with the results from the pillar testing experiments, while doublecantilever testing of the CAE-PVD CrN coatings gave a fracture toughness of $1.75 \mathrm{MPa} \mathrm{m}{ }^{1 / 2}$, which is roughly $25 \%$ lower than the results obtained by pillar splitting [23]. It is worth noting that the values of the standard deviations obtained by the different techniques are also similar, thus suggesting that the same microstructural features affect the experimental results on the samples and that the explanation for the observed discrepancies might be found in a systematic bias given by the specific calibration procedures for each method.

Results for the BMG sample are given in Fig. 10. As clearly visible from both the SEM image of a deformed pillar and the corresponding load-displacement curve, neither crack nucleation nor unstable propagation was observed. This result was confirmed for a range of pillar diameters between 2 and 15 microns, all with an aspect ratio fixed at 1.0. Deformation in the BMG samples occurred mostly by propagation of discrete shear bands that are visible as a series of serrations in the load-displacement curve of Fig. 10(b). This type of deformation is common in BMGs [48]. 


\section{Discussion}

The results in this work compare several techniques for measuring fracture toughness using micro-scale specimens. On one hand, good agreement is found between measurements obtained by single-cantilever bending and pillar splitting experiments on the MS-PVD CrN coating. On the other hand, a 25\% discrepancy (which still represents a reasonable agreement) is found between the results obtained by double-cantilever tests and those obtained by the indentation pillar splitting technique on the CAE-PVD CrN coating. For the pillar splitting method, tests on a bulk silicon wafer give fracture toughnesses that are in the range reported in the literature [48].

In a recent paper [27], a comparison is made among the pillar splitting, the single and clamped cantilever on $\mathrm{Si}(100)$. A value of $0.75 \mathrm{MPa} \cdot \mathrm{m}^{1 / 2}$ is found by pillar splitting experiments using a Cube-Corner indenter. However, the gamma coefficient that is used in that work was calculated for the case of a Berkovich indenter. According to previous studies [16-17], one can expect a larger gamma coefficient for a Cube-Corner indenter and consequently a higher toughness value, probably close to $1.0 \mathrm{MPa} \cdot \mathrm{m}^{1 / 2}$ and in good accordance with the one that was measured in the present work.

Several points can be raised to explain the observed discrepancies among the different methods. All are generally related to the fact that the methods require calibration procedures to obtain a numerical coefficient needed for toughness calculation. More specifically, errors in toughness for pillar indentation splitting may arise from deviations between the idealized, isotropic, elastic-perfectly plastic finite element simulations and the often columnar grained microstructure of PVD films. PVD coatings also often exhibit growth defects that may facilitate crack nucleation/propagation. In addition, the taper angle of the pillar, tip rounding of the Berkovich indenter tip, and similar minor geometric imperfections were not taken into account in the simulations. Taper angle is usually a significant issue only for pillars whose diameters are less than $1 \mu \mathrm{m}$. In the present work, the diameter of pillars was in the range of 3-6 $\mu \mathrm{m}$, so taper angle influences are probably not large.

Ion damage caused by FIB milling is expected to be very different for the testing techniques, with FIB damage leading to local changes of the mechanical properties. For pillar splitting, crack nucleation/propagation occurs in a volume of material that is relatively far from the FIB milled surfaces and is thus mostly unaffected by the FIB damage. In contrast, crack propagation in the single- and double-cantilever tests occurs directly in the FIB affected zone at the ion milled crack tip. Useful strategies to reduce ion-damage effects in case of cantilever or notched beam geometries have been developed and should be employed in these situations [50-51]. The friction between the flat-punch indenter and the sample can have a significant effect on fracture toughness measurements in case of the double-cantilever method [23], but its influence on the pillar splitting tests has not yet been evaluated. Overall, both the FIB milled micro-cantilever and pillar indentation 
splitting measurements of fracture toughness are generally in good agreement considering the uncertainties and assumptions made in the measurement procedures.

It is important to note that the compressive residual stress in the films could play an important role in determining their in-service failure modes. Noting that the pillar splitting method gives the stress-free fracture toughness, it is possible to calculate the apparent toughness of a residually stressed coating from [12]:

$$
K_{C}=K_{C}^{0}+Z \cdot \sigma_{R} \cdot c^{1 / 2},
$$

where $\sigma_{R}$ is the absolute value of the compressive surface residual stress, $\boldsymbol{c}$ is the radial dimension of the crack, $\boldsymbol{K}_{\boldsymbol{C}}{ }_{\boldsymbol{C}}$ is the fracture toughness that would be observed in absence of residual stress, and $\boldsymbol{Z}$ is a crack-geometry parameter [12] which is equal to 1.26 when the radial dimension of the crack is equal to its depth (i.e., a semi-circular crack). As reported in a previous paper [26], Eq. 8 is very useful as an estimation of the apparent toughness of thin films in the presence of compressive residual stresses.

Results on the BMG sample showed no unstable failure or cracking during pillar indentation. This observation leads to the question of whether the pillar splitting method might be useful in testing metallic or polymeric systems where the hardness is often much lower and the fracture toughness much greater than in ceramic or glassy materials. For such materials, it is not clear whether cracks will nucleate during pillar splitting tests. The cohesive zone finite element methods used here describe energy dissipation during equilibrium crack growth. That the same mathematical model can describe crack nucleation is unlikely, especially in the complex stress and deformation environment of indentation. To aid in the discussion of testing metals/polymers, we consider three different scenarios for crack nucleation: (1) crack nucleation at the theoretical cohesive strength; (2) crack initiation from pre-existing flaws [43]; and (3) crack nucleation induced by plastic deformation in the vicinity of the indentation contact [44]. From a continuum viewpoint, the maximum tensile stresses that develop during indentation are limited by the yield strength, and thus crack nucleation at the theoretical strength is not possible [45]. In cases 2 and 3, models have been proposed by Lawn and Evans [43] and Hagan [44] in which the load for crack initiation, $P_{\text {init }}$, during Vickers indentation is given by:

$$
P_{i n i t}=\chi \frac{K_{I c}^{4}}{H^{3}},
$$

where the coefficient $\chi$ was for a Vickers indenter has a value of about $2.2 \times 10^{4}$. We note that differences are to be expected between the four-sided Vickers and three-sided Berkovich used in the current experiments. 
In light of this, it is important to consider the diameter of the pillar required to achieve splitting during testing. Assuming that Eq. 9 holds, the length of the diagonal of a Vickers indent in a material at the crack initiation load is given by:

$$
d=\sqrt{2 \chi} \frac{K_{I}^{2}}{H^{2}}
$$

Given that the pillar diameter must be at least as big as the size of the hardness impression at crack initiation, minimum pillar diameter contours predicted from Eq. 10 are shown in Fig. 11 as a function of $\log _{10} \boldsymbol{K}_{I c}$ and $\log _{10} \boldsymbol{H}$ for a range of hardness from $1 \mathrm{GPa}$ to $40 \mathrm{GPa}$ and fracture toughnesses between $0.6 \mathrm{MPa} \mathrm{m}^{1 / 2}$ and $25 \mathrm{MPa} \mathrm{m}^{1 / 2}$. These ranges cover most brittle materials as well as the harder and more brittle metals. The plot shows that there is a strong dependence on the ratio of fracture toughness to hardness, as predicted from Eq. 10. In addition, data points representing the location of the $\mathrm{TiN}$ and $\mathrm{CrAlN} / \mathrm{Si}_{3} \mathrm{~N}_{4}$ pillar materials examined in a previous study have been added to Fig. 11 [26]. The experiments in those cases used pillar diameters of greater than $\sim 3 \mu \mathrm{m}$, well above predicted requirement of $\sim 1 \mu \mathrm{m}$.

For comparison, it is useful to consider a glass-like material with $\boldsymbol{H}=9 \mathrm{GPa}$ and $\boldsymbol{K}_{\boldsymbol{I c}}=1.0$ $\mathrm{MPa} \mathrm{m}{ }^{1 / 2}$, and a brittle metal-like material with $\boldsymbol{H}=2 \mathrm{GPa}$ and $\boldsymbol{K}_{\boldsymbol{I c}}=10 \mathrm{MPa} \mathrm{m}{ }^{1 / 2}$. According to Hagan's model, the lower bound estimate results in crack initiation loads of $\sim 1.2 \times 10^{-3} \mathrm{~N}$ and $\sim 1100$ $\mathrm{N}$ for the glass-like and metal-like materials, respectively. The corresponding Vickers diagonal lengths are $\sim 0.50 \mu \mathrm{m}$ and $\sim 1050 \mu \mathrm{m}$. Assuming that the yield strength of the materials is $\sim \boldsymbol{H} / 3$ [46], then the crack length criterion for small scale yielding (i.e., $c>2.5 K_{I c}{ }^{2} / Y^{2}$ ) from [40] for these materials is $\sim 0.100 \mu \mathrm{m}$ and $\sim 560 \mu \mathrm{m}$, respectively. The smallest diameter pillar for the estimation of fracture toughness is then the sum of the Vickers diagonal and two times the minimum crack length, which for our example materials is $\sim 1 \mu \mathrm{m}$ and $\sim 2200 \mu \mathrm{m}$. The glass-like and metal-like materials described above have been added to Fig. 11 for comparison. A similar analysis for a brittle polymer (e.g., PMMA with $\boldsymbol{H}=0.2 \mathrm{GPa}$ and $\boldsymbol{K}_{\boldsymbol{I} \boldsymbol{c}}=1 \mathrm{MPa}^{1 / 2}$ [47]) results in a minimum pillar diameter of the same order of magnitude as the metal-like material.

While this analysis is based on a number of assumptions, the end result is that testing of a metallic or polymeric material would require loads that are $\sim 5$ orders of magnitude greater than for ceramic materials and very large diameter pillars. This then necessarily means that small-scale testing of these materials by the pillar splitting method is not possible. We also note that stable crack growth is observed before failure in the pillar splitting test, and, thus, toughness values from this measurement in metallic materials may be different from standard toughness tests due to the possibility of rising R-curve behavior.

The requirements for crack nucleation in the pillar splitting technique provide a natural segue into the future of indentation testing on pillars of brittle materials. While the importance of 
fracture toughness cannot be ignored, the micromechanics describing crack nucleation during contact likely plays an important role in determining the strength and reliability of brittle coatings. Techniques that examine the onset of cracking, flaw distributions, and mechanisms of plastic deformation may lead to the development of more robust protective coatings for future applications. Experimental results in combination with simulations that identify unstable conditions may also aid in designing materials and structures for more damage tolerant coatings. 


\section{Conclusion}

In this work, a comparison has been made of indentation pillar splitting and cantilever-based techniques for small-scale fracture toughness measurement. Using cohesive zone finite element techniques to simulate indentation pillar splitting, the relationship among fracture toughness, critical load, and pillar radius has been quantified for a wide range of $\boldsymbol{E} / \boldsymbol{H}$. Explanations for the origins of the proposed equations relating the splitting load to the fracture toughness are also given, and the effects of substrate compliance on the results are quantified.

The new experimental results show good agreement between the single cantilever and pillar splitting methods for the $\mathrm{CrN}$ thin film material, while a reasonable agreement (within $25 \%$ ) is found between the double-cantilever and the pillar splitting techniques. Discrepancies among the methods are possibly caused by systematic errors in the calibration procedures required for each. The pillar indentation method takes advantage of the simplicity of indentation testing, no post-test measurements are required, and both FIB damage and residual stress influences are minimized. However, a critical pillar radius for crack nucleation has been identified that makes the pillar splitting method impractical for most metals. Thus, for metallic specimens, the notched cantilever techniques are likely to be preferred. It is concluded that both the micro-cantilever and pillar splitting techniques are relatively reliable and straightforward methods for micro-scale assessment of fracture toughness of ceramics, provided that the main assumptions of the methods can be verified. 


\section{Acknowledgements}

The research activities of M. Sebastiani were funded by the Fulbright Scholar Program, through the appointment of a Fulbright Research Scholarship by the US-Italy Fulbright Commission. The cohesive zone finite element simulations were performed under the support of NSF grant number CMMI 0926798, and the pillar cracking experiments under support of the U.S. Department of Energy, Office of Basic Energy Sciences, Materials Sciences and Engineering Division. FIB sample preparation was performed at the interdepartmental laboratory of electron microscopy (LIME lab) of Roma TRE University, with the assistance of Daniele de Felicis. The authors acknowledge Shiyu Liu, William Clegg (Univ. of Cambridge) and Johann Michler (EMPA) for providing the CAE-PVD CrN samples, Rostislav Daniel (University of Leoben) for providing the MS-PVD CrN sample, and Easo P. George (formerly of Oak Ridge National Laboratory; now at Ruhr University Bochum) for production of the BMG sample. 


\section{References}

[1].W.C. Oliver and G.M. Pharr, J. Mater. Res. 7 (1992) p.8.

[2].W.C. Oliver and G.M. Pharr, J. Mater. Res. 19 (2004) p.3.

[3].E.G. Herbert, W.C. Oliver, A. Lumsdaine, G.M. Pharr, J. Mater. Res. 24 (2009) p.626.

[4].V. Maier, K. Durst, J. Mueller, B. Backes, H. Werner Höppel, M. Göken, J. Mater. Res. 26 (2011) p.1421

[5].E. G. Herbert, W. C. Oliver, G. M. Pharr, Phil. Mag. 86 Nos. 33-35 (2006) p.5521

[6].M. Sebastiani, E. Bemporad, F. Carassiti and N. Schwarzer, Phil Mag. 91 (2011) p.1121

[7].A. A Volinsky, J B Vella, W W Gerberich, Thin Solid Films 429 (2003) p.201

[8].B.R. Lawn and T.R. Wilshaw, J Mater Sci. 10 (1975) p.1049.

[9].A.G. Evans and E.A. Charles, J Am Ceram Soc. 59 (1976) p.371.

[10]. D.B. Marshal and B.R. Lawn, J Mater Sci. 14 (1979) p.2001.

[11]. B.R. Lawn, A.G. Evans and D.B. Marshall. J Am Ceram Soc. 63 (1980) p.574.

[12]. G.R. Anstis, P. Chantikul, B.R. Lawn, D.B. Marshall, J Am Ceram Soc. 64 (1981) p.533.

[13]. M. T. Laugier, J Mat Sci Lett. 6 (1987) p.355.

[14]. R.F. Cook, G.M. Pharr, J Am Ceram Soc 73 (1990) p.787.

[15]. K.I. Schiffmann, Phil Mag. 91 (2011) p. 1163.

[16]. J. H. Lee, Y. F. Gao, K. E. Johanns and G. M. Pharr, Acta Mater. 60 (2012) p.5448.

[17]. K. E. Johanns, J. H. Lee, Y. F. Gao, and G. M. Pharr, Modeling Simul Mater Sci Eng. 22 (2014) 015011.

[18]. D. Di Maio and S.G. Roberts, J. Mater. Res. 20 (2005) p.299

[19]. B. N. Jaya, V. Jayaram, Int J Fract (2014) 188, p.213

[20]. A. Zeilinger, R. Daniel, M. Stefenelli, B. Sartory, L. Chitu, M. Burghammer, T. Schöberl, O. Kolednik, J. Keckes and C. Mitterer, Mechanical Property Enhancement in Laminates through Control of Morphology and Crystal Orientation, Acta Mater. (2014) submitted

[21]. D. Kupka , N. Huber, E.T. Lilleodden, Journal of the Mechanics and Physics of Solids 64 (2014) p.455

[22]. J. Schaufler, C. Schmid, K. Durst, M. Göken, Thin Solid Films 522 (2012) p.480

[23]. S. Liu, J. M. Wheeler, P.R. Howie, X.T. Zeng, J. Michler and W.J. Clegg, App Phys Lett. 102 (2013) p.171907.

[24]. H. D. Espinosa, B. Peng, Journal of Microelectromechanical Systems, 14(1) (2005), p. 153

[25]. B. Merle, M. Goken, Acta Materialia 59 (2011) p.1772

[26]. M. Sebastiani, K.E. Johanns, E.G. Herbert, F. Carassiti, G.M. Pharr, Phil Mag. 2014, http://dx.doi.org/10.1080/14786435.2014.913110

[27]. B. N. Jaya, C. Kirchlechner, G. Dehm, J. Mater. Res., Vol. 30, No. 5, Mar 14, 2015 
[28]. Simulia, ABAQUS User's Manual. 2008: Simulia Co.

[29]. A. Muchtar, L.C. Lim and K.H. Lee, J Mat Sci. 38 (2003) p.235

[30]. W. Zhang and G. Subhash, Int J Sol Struct. 38 (2001) p.5893

[31]. A. Cornec, I. Scheider and K.-H. Schwalbe, Eng Fract Mech. 70 (2003) p.1963

[32]. H. Wan, Y. Shen, Q. Chen and Y. Chen, J Mat Res. 25 (2010) p. 2224

[33]. Y.F. Gao and A.F. Bower, Model Simul Mater Sci. 12 (2004) p.453

[34]. A.M. Korsunsky, M. Sebastiani and E. Bemporad, Surf Coat Technol. 205 (2010) p.2393

[35]. M. Sebastiani, C. Eberl, E. Bemporad and G. M. Pharr, Mater Sci Eng A. 528 (2011) p.7901

[36]. S. Suresh and A.E. Giannakopoulos, Acta Mater. 46 (1998) p.5755

[37]. Tada, H., P.C. Paris, and G.R. Irwin, The stress analysis of cracks handbook. 1973: Del Research Corp

[38]. Shah, R.C. and A.S. Kobayashi, International Journal of Fracture, 1973. 9(2): p. 133

[39]. Giannakopoulos, A.E., P.L. Larsson, and R. Vestergaard, 1994. 31(19): p. 2679

[40]. Larsson, P.L., et al., International Journal of Solids and Structures, 1996. 33(2): p. 221

[41]. Anderson, T.L., Fracture Mechanics: Fundamentals and Applications. 2005: Taylor \& Francis

[42]. Saha, R. and W.D. Nix, Effects of the substrate on the determination of thin film mechanical properties by nanoindentation. Acta Materialia, 2002. 50(1) p. 23

[43]. Lawn, B.R. and A.G. Evans, A model for crack initiation in elastic/plastic indentation fields. Journal of Materials Science, 1977. 12(11): p. 2195

[44]. Hagan, J.T., Micromechanics of crack nucleation during indentations. Journal of Materials Science, 1979. 14(12): p. 2975

[45]. Johnson, K.L., Contact Mechanics. 1987: Cambridge University Press

[46]. Tabor, D., The Hardness of metals. 1951, Oxford: Clarendon Press

[47]. Choi, S.R. and J.A. Salem, Fracture toughness of PMMA as measured with indentation cracks. Journal of Materials Research, 1993. 8(12): p. 3210

[48]. R. Ballarini, R. L. Mullen, Y. Yin, H. Kahn, S. Stemmer, A. H. Heuer, J. Mater. Res., Vol. 12, No. 4, Apr 1997, p. 915

[49]. F. Haag, D. Beitelschmidt, J. Ecker, K. Durst, Acta Materialia 70 (2014) p. 188

[50]. K. Matoy, T. Detzel, M. Müller, C. Motz , G. Dehm, Surface \& Coatings Technology 204 (2009) 878-881

[51]. K. Matoy, H. Schönherr, T. Detzel, T. Schöberl, R. Pippan, C. Motz, G. Dehm, Thin Solid Films 518 (2009) 247-256

[52]. E. Bemporad, C. Pecchio, S. De Rossi, F. Carassiti, Surface \& Coatings Technology 188189 (2004) 319- 330 


\section{Tables}

Table 1 - Material properties and experimental results.

\begin{tabular}{|c|c|c|c|c|}
\hline Material & (100) Silicon & MS-PVD CrN & CAE-PVD CrN & $\begin{array}{c}\text { Zr-Cu-Ni-Ti-Al } \\
\text { BMG sample }\end{array}$ \\
\hline Film Thickness, $t(\mu \mathrm{m})$ & N/A & 3.0 & 9.0 & N/A \\
\hline $\begin{array}{c}\text { Elastic Modulus, } E \\
\text { (GPa) }\end{array}$ & $135.2 \pm 4$ & $320 \pm 15$ & 342.3 & $105.8 \pm 0.7$ \\
\hline FE Poisson's Ratio, $v$ & 0.25 & 0.25 & 0.25 & 0.30 \\
\hline $\begin{array}{c}\text { Exp. Hardness, } H \\
\text { (GPa) }\end{array}$ & $9.26 \pm 0.5$ & $18.5 \pm 0.5$ & 21.84 & $6.70 \pm 0.05$ \\
\hline $\begin{array}{c}\text { FE Yield Strength, } Y \\
(\text { (GPa) }\end{array}$ & 4.0 & 7.75 & 9.37 & N/A \\
\hline FE $\gamma($ Eq. 5) & 0.280 & 0.313 & 0.286 & N/A \\
\hline Pillar Radius, $R(\boldsymbol{\mu m})$ & $2.35 \pm 0.1$ & $1.1 \pm 0.1$ & $3.3 \pm 0.1$ & $2.5 \pm 0.1$ \\
\hline $\begin{array}{c}\text { Instability load, } P_{c} \\
(\mathrm{mN})\end{array}$ & $15.00 \pm 1.58$ & $11.52 \pm 0.38$ & $45.8 \pm 1.7$ & $\begin{array}{l}\text { NO instability } \\
\text { observed }\end{array}$ \\
\hline $\mathrm{K}_{c}($ this study, MPa $\sqrt{ } \mathbf{m})$ & $1.16 \pm 0.12$ & $3.13 \pm 0.10$ & $2.19 \pm 0.08$ & N/A \\
\hline $\begin{array}{c}\mathrm{K}_{c} \text { (independent } \\
\text { measurements, } \\
\text { MPa } \sqrt{ } \mathrm{m})\end{array}$ & $0.9-1.2 *$ & $3.10 \pm 0.2 * *$ & $1.75 \pm 0.07 * * *$ & N/A \\
\hline
\end{tabular}

*Data from literature [47]

**Data obtained on the SAME sample by the single-cantilever bending test [20]

***Data obtained on the SAME sample by the double-cantilever method [23] 


\section{List of figure captions}

Figure 1. Radial cracks originating at the edges of a Berkovich indentation for a titanium nitride (TiN) coating material (SEM, 5kV, ETD SE).

Figure 2. Schematic representation of the micro-cantilever bending geometry (reproduced from [18] with permission of the authors).

Figure 3. Schematic representation of the double-cantilever bending geometry (reproduced from [23] with permission of the authors).

Figure 4. An example of a pillar after splitting [26].

Figure 5. The finite element mesh used in the simulations of pillar indentation fracture. A 1/6th symmetric model for a Berkovich indenter is used, with the pillar height greater than its diameter.

Figure 6. Representative plots of load as a function of indenter displacement for the same representative material, whose properties are shown on bottom-right of the figure. Three different coating/substrate combinations are reported in order to show the effects of substrate compliance on the critical splitting load.

Figure 7. Crack geometries in a representative pillar splitting test just before and just after the instability load.

Figure 8. Calculated values of $\boldsymbol{\gamma}$ coefficients (Eq. 5) for a wide range of $\boldsymbol{E} / \boldsymbol{H}$ values and several coating/substrate combinations.

Figure 9. SEM micrographs of pillars and representative load-displacement curves obtained from the three ceramic materials under investigation: (a,b) single crystal (100) silicon; (c,d) CAE-PVD $\mathrm{CrN}$, where a top-view of the pillar after splitting is shown; and (e,f) MS-PVD CrN.

Figure 10. (a) SEM micrograph and (b) the corresponding load-displacement curve for indentation of a bulk metallic glass pillar. No crack nucleation was observed during the test. The serrations in the load-displacement curve are due to the propagation of discrete shear bands.

Figure 11. Contours predicted from Eq. 10 for the minimum pillar diameter required to produce unstable crack propagation during pillar indentation testing as a function of $\log _{10} K_{I c}$ and $\log _{10} H$. Hardnesses range from $1 \mathrm{GPa}$ to $40 \mathrm{GPa}$ and fracture toughnesses from $0.6 \mathrm{MPa} \mathrm{m}{ }^{1 / 2}$ to $25 \mathrm{MPa}$ $\mathrm{m}^{1 / 2}$. 


\section{Figures}

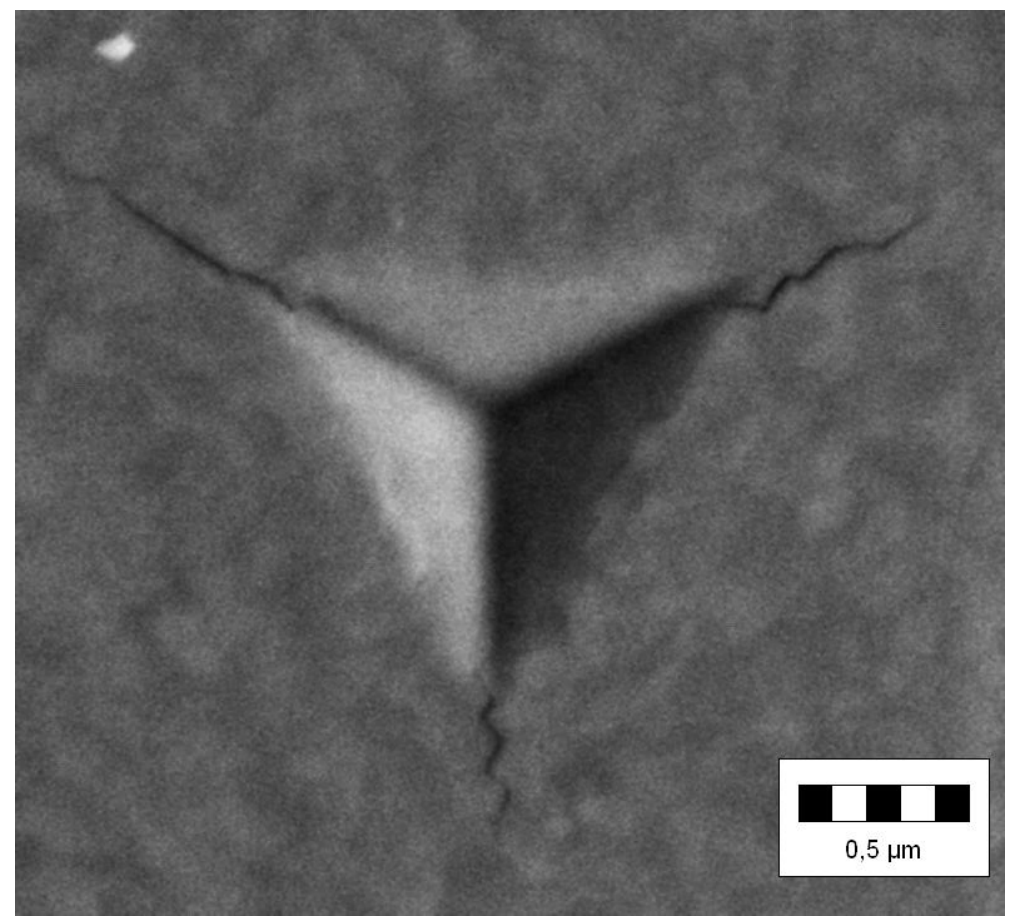

Figure 1. Radial cracks originating at the edges of a Berkovich indentation for a titanium nitride (TiN) coating material (SEM, 5kV, ETD SE). 


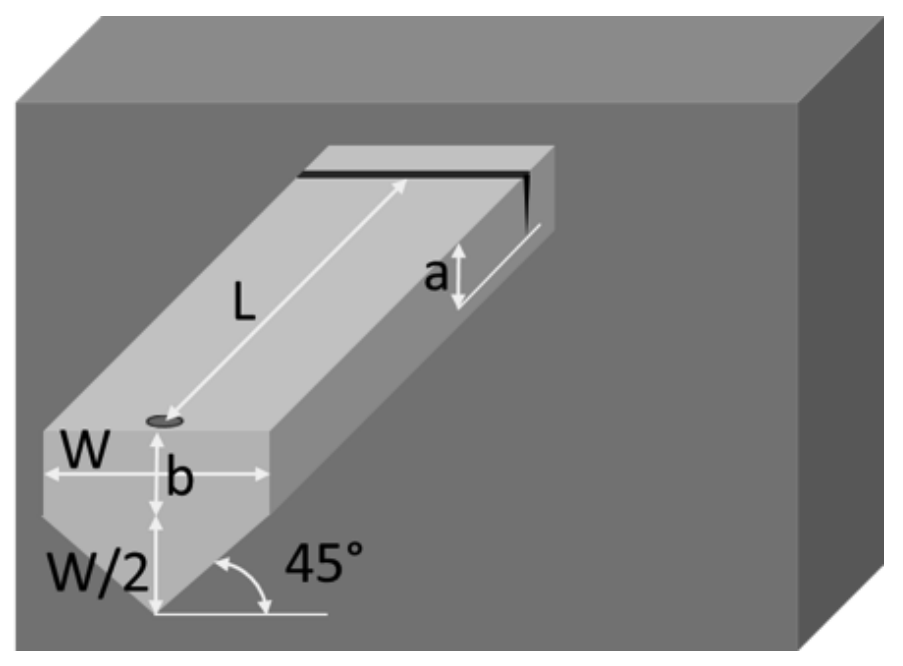

Figure 2. Schematic representation of the micro-cantilever bending geometry (reproduced from [18] with permission of the authors). 


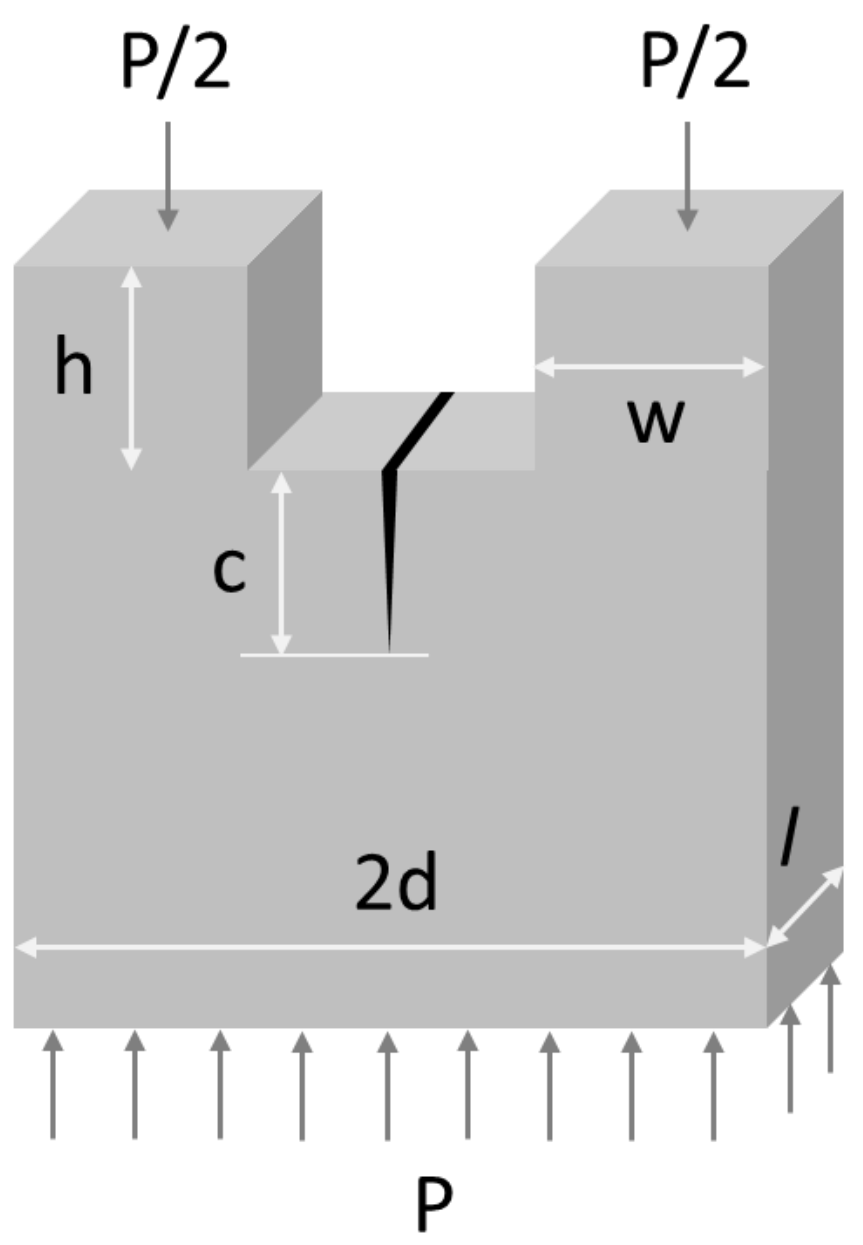

Figure 3. Schematic representation of the double-cantilever bending geometry (reproduced from [23] with permission of the authors). 


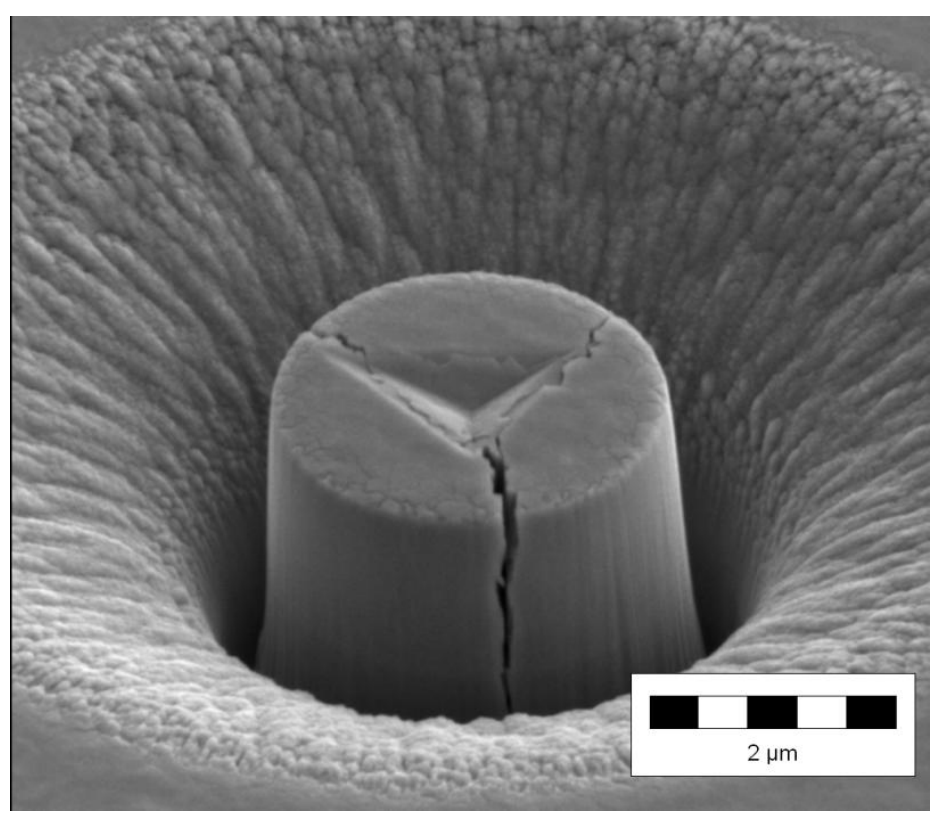

Figure 4. Example of a pillar after splitting [26] 


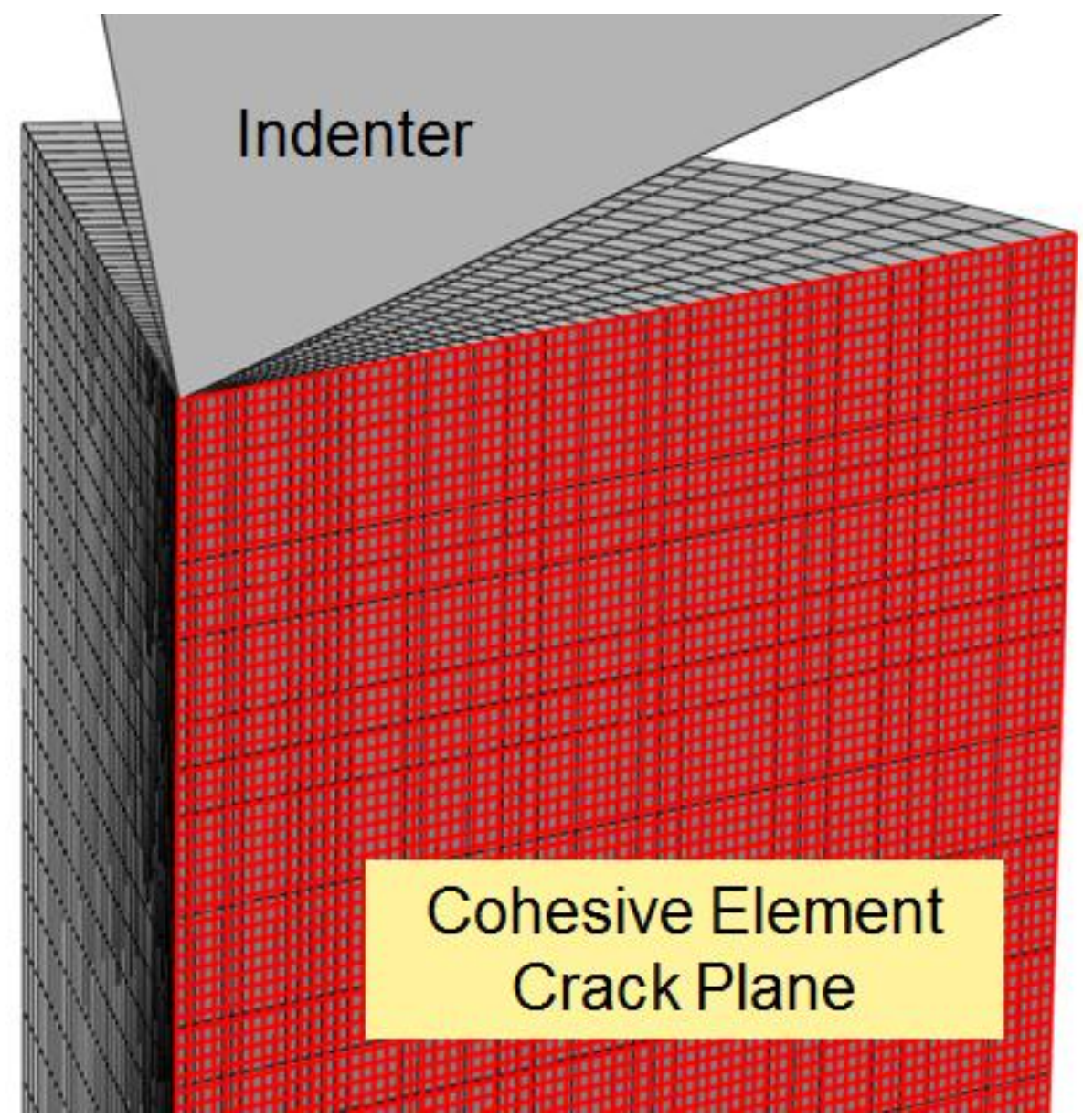

Figure 5. The finite element mesh used in the simulations of pillar indentation fracture. A 1/6th symmetric model for a Berkovich indenter is used, with the pillar height greater than its diameter. 


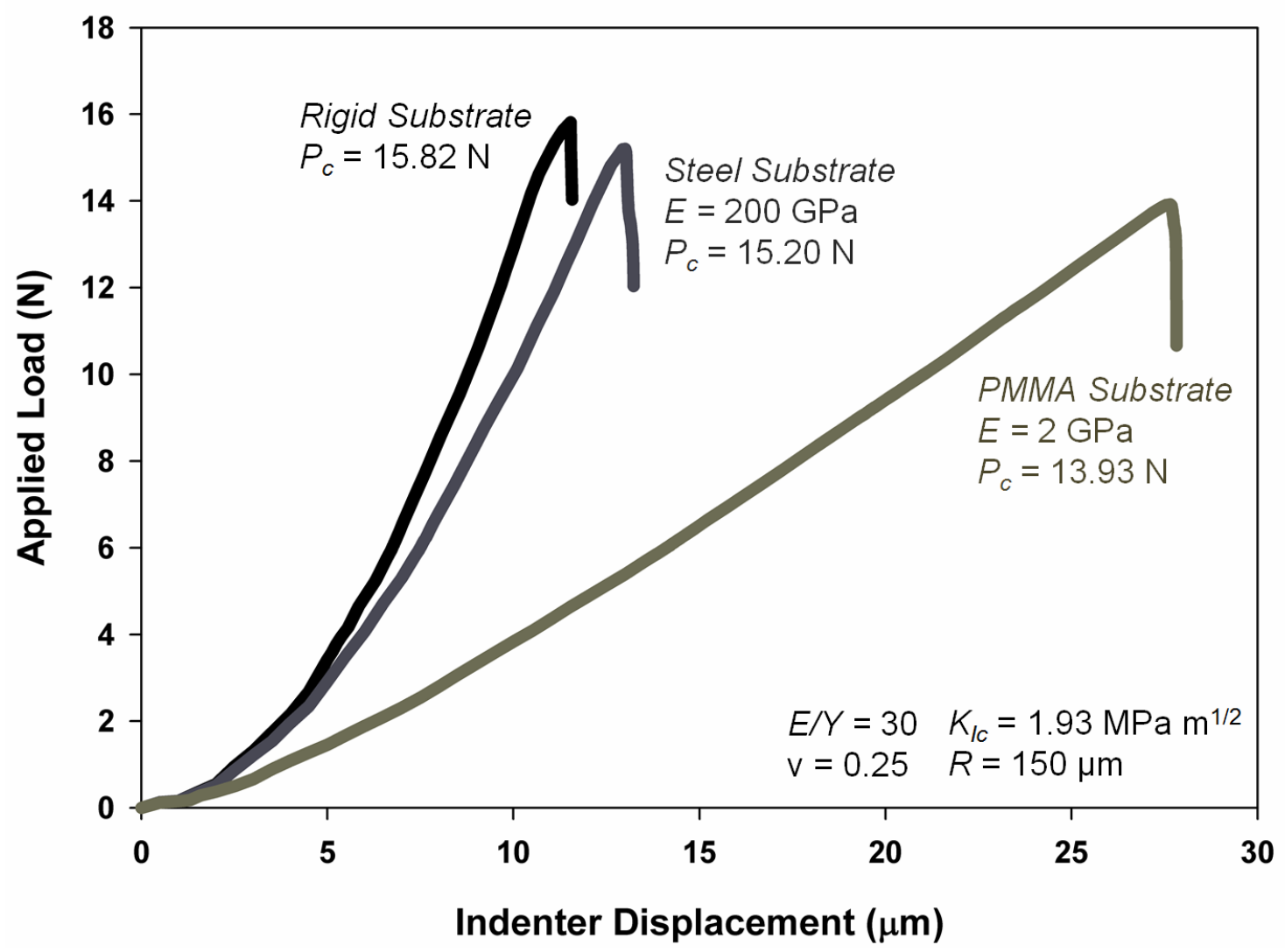

Figure 6. Representative plots of load as a function of indenter displacement for the same representative material, whose properties are shown on bottom-right of the figure. Three different coating/substrate combinations are reported in order to show the effects of substrate compliance on the critical splitting load. 


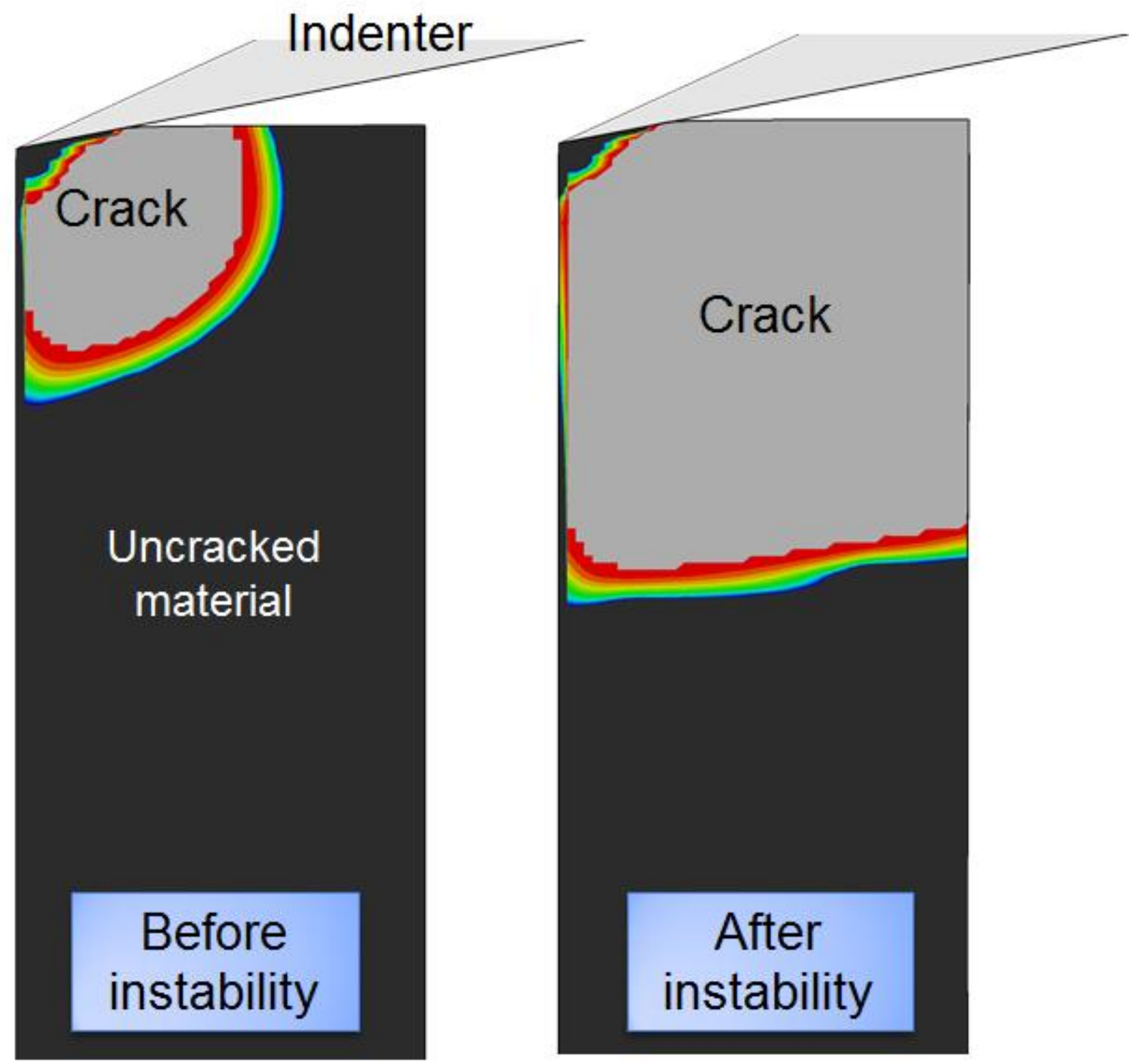

Figure 7. Crack geometries in a representative pillar just before and just after the instability load. 


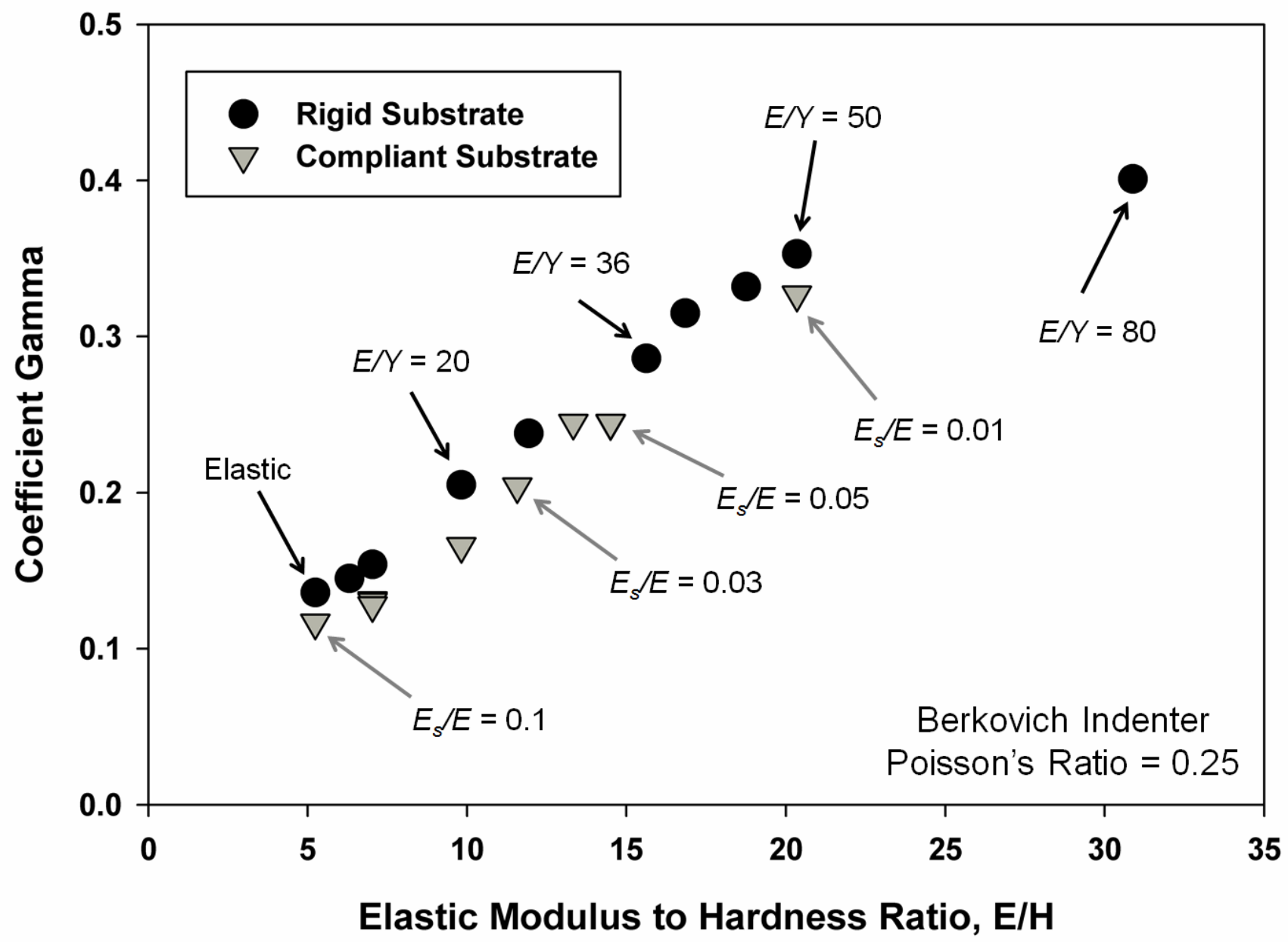

Figure 8. Calculated values of $\gamma$ coefficients (Eq. 5) for a wide range of E/H values and several coating/substrate combinations. 


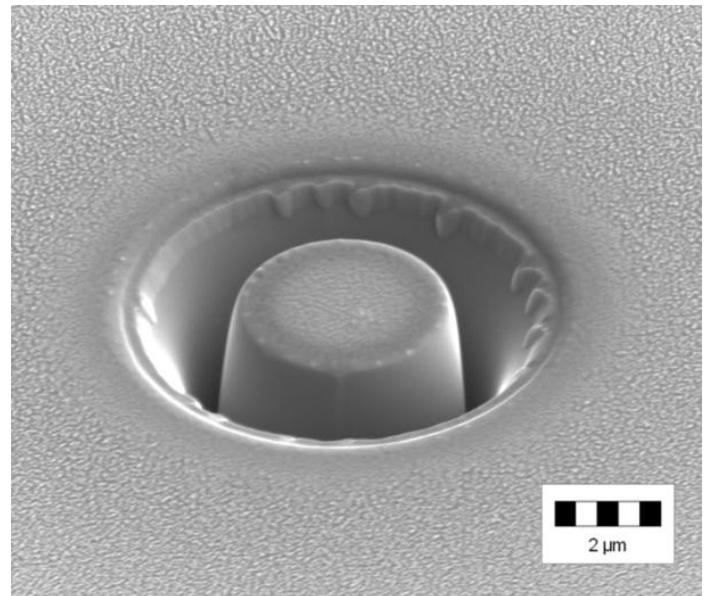

(a)

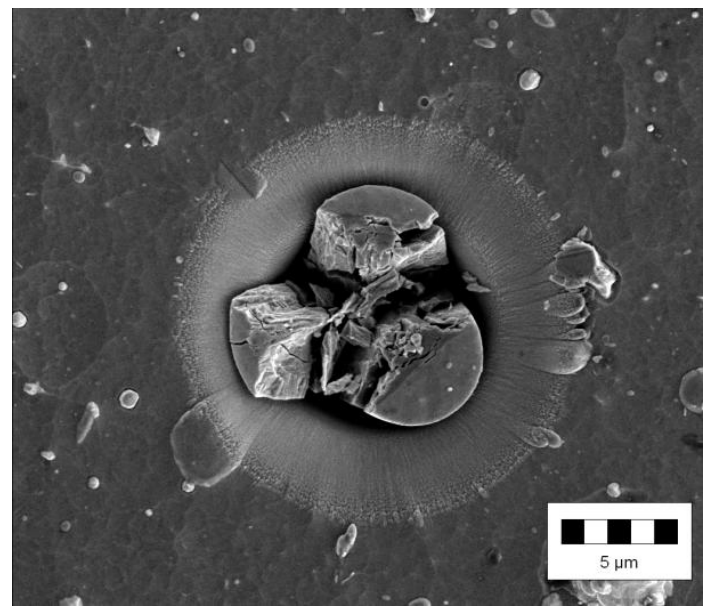

(c)

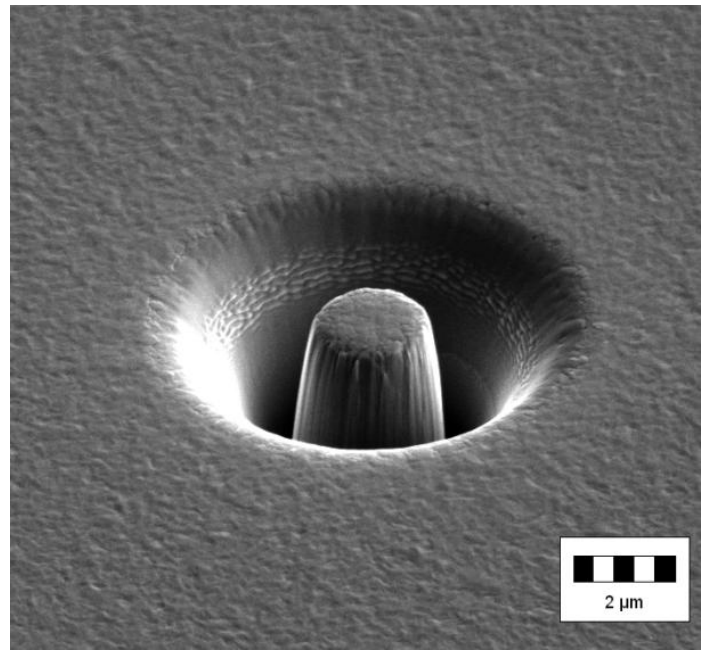

(e)

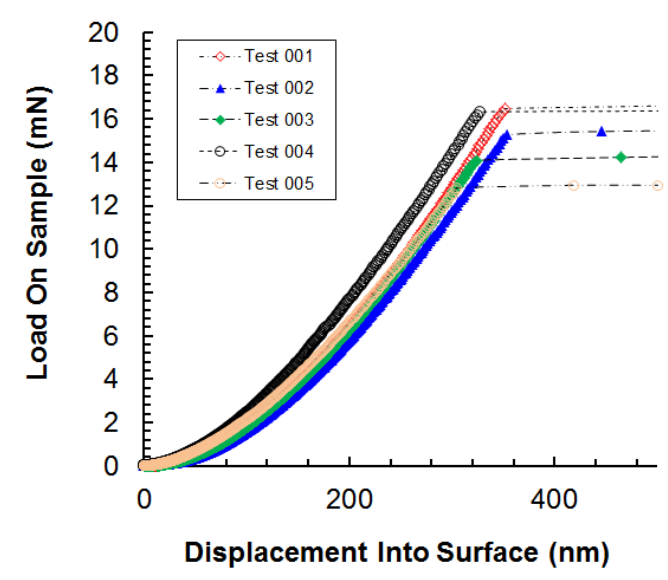

(b)

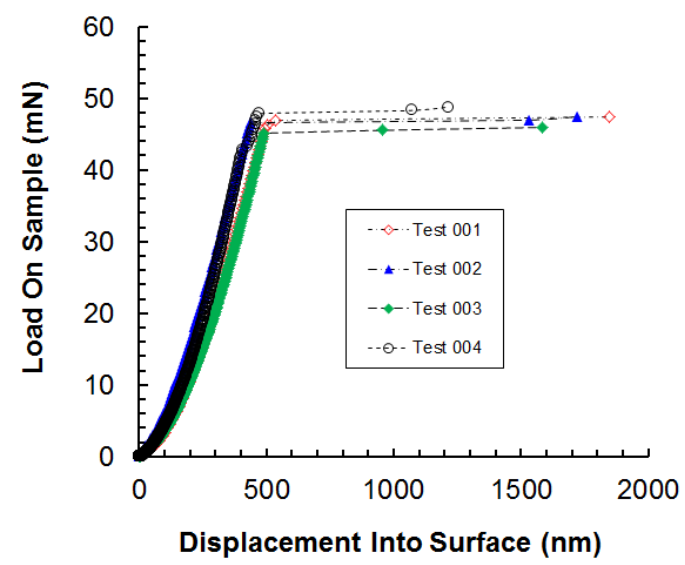

(d)

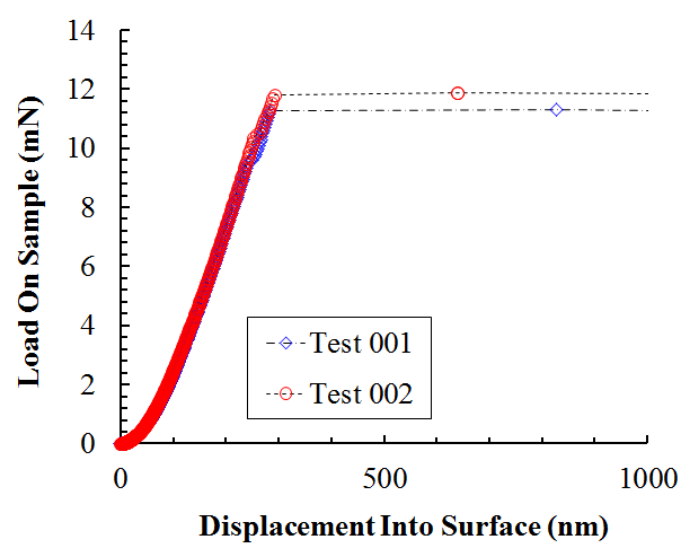

(f)

Figure 9. SEM micrographs of pillars and representative load-displacement curves obtained from the three ceramic materials under investigation: (a,b) single crystal (100) silicon; (c,d) CAE-PVD CrN, where a top-view of the pillar after splitting is shown; and (e,f) MS-PVD $\mathrm{CrN}$. 


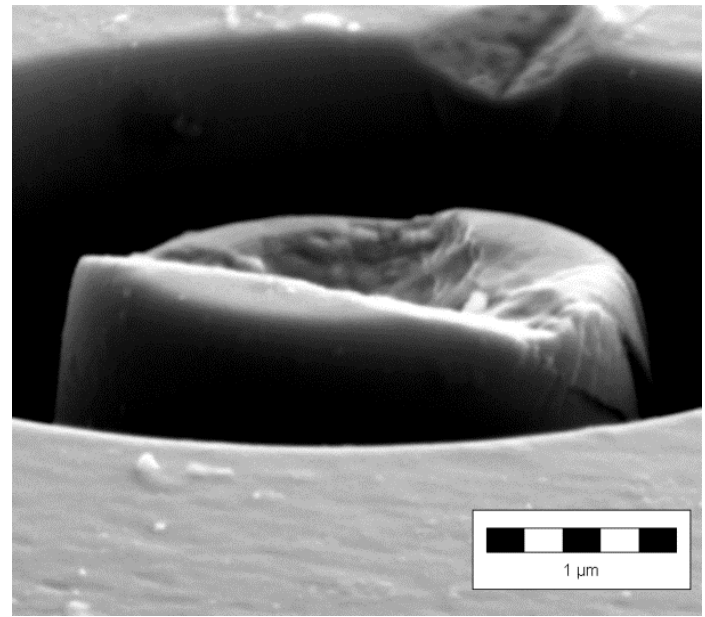

(a)

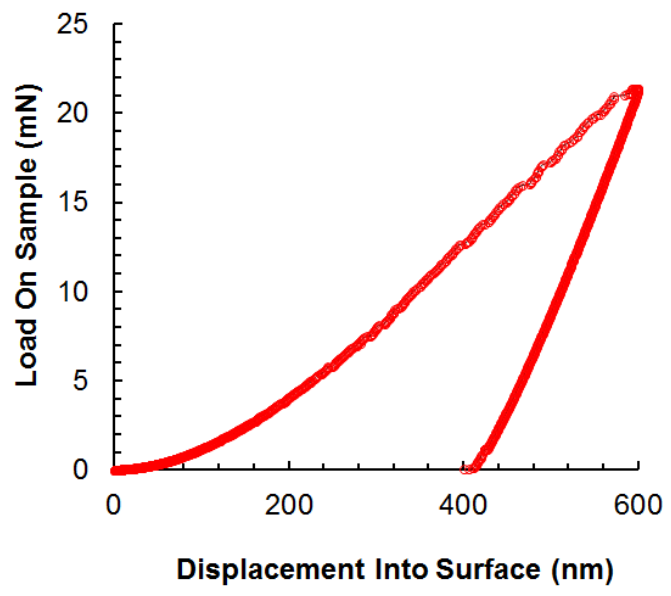

(b)

Figure 10. (a) SEM micrograph and (b) the corresponding load-displacement curve for indentation of a bulk metallic glass pillar. No crack nucleation was observed during the test. The serrations in the load-displacement curve are due to the propagation of discrete shear bands.. 


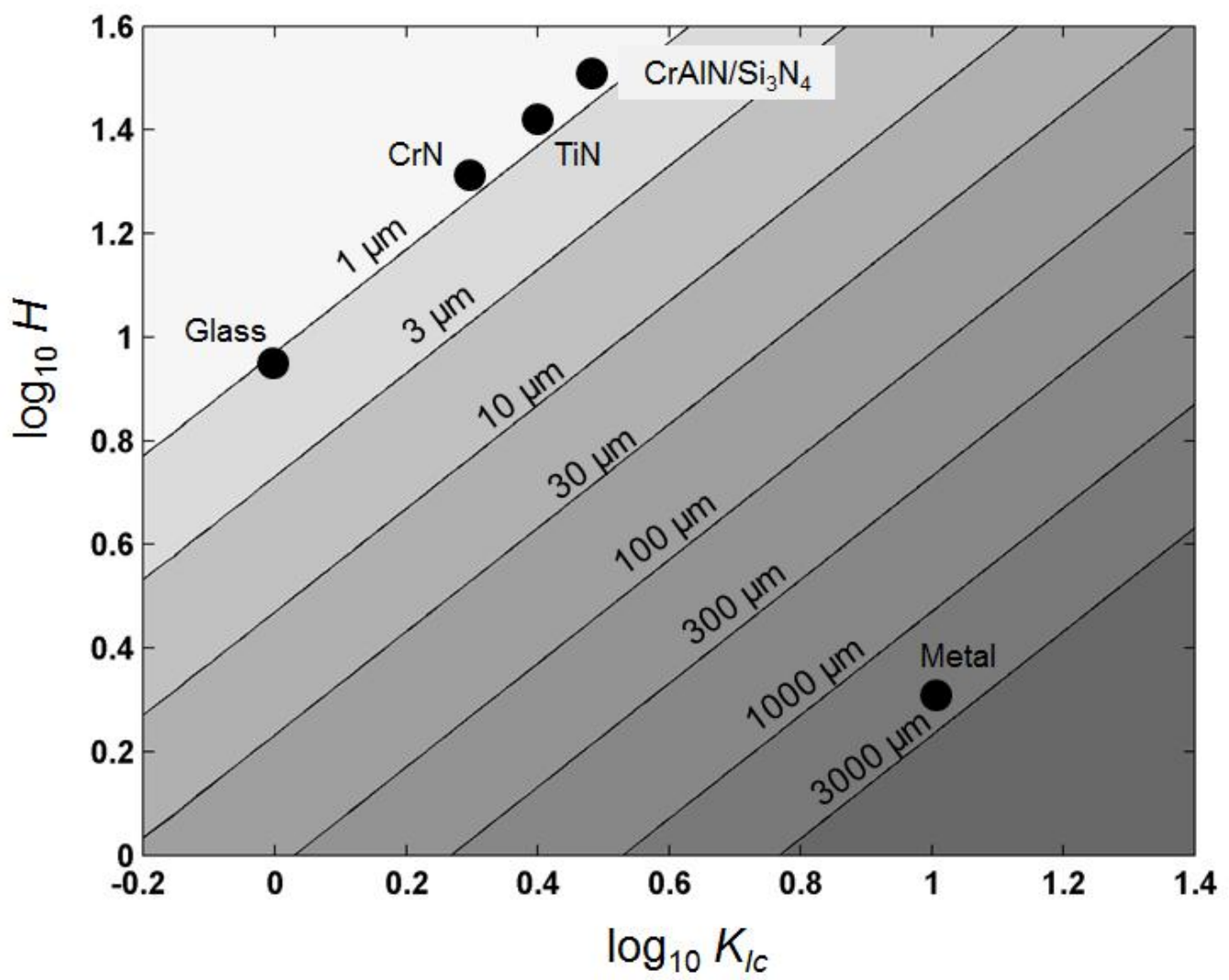

Figure 11. Contours predicted from Eq. 10 for the minimum pillar diameter required to produce unstable crack propagation during pillar indentation testing as a function of $\log _{10} K_{I c}$ and $\log _{10} \mathrm{H}$. Hardnesses range from $1 \mathrm{GPa}$ to $40 \mathrm{GPa}$ and fracture toughnesses from 0.6 MPa $\mathrm{m}^{1 / 2}$ to $25 \mathrm{MPa} \mathrm{m}^{1 / 2}$. 